\title{
A centralized reverse channel structure with flexible manufacturing under the stock out situation
}

\author{
S.R. Singh ${ }^{a}$, Leena Prasher ${ }^{b}$ and Neha Saxena ${ }^{a^{*}}$
}

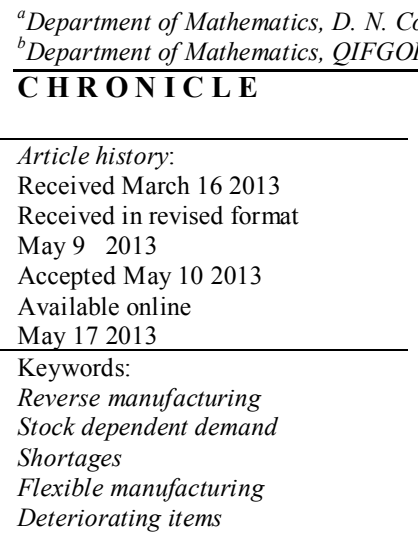

\section{Introduction}

Environmental degradation has emerged as a serious social and economic problem. In fact, several governmental policies also encourage the business organizations to re-use or re-cycle used materials with a view to prevent further environmental degradation. The impact of this consciousness on organizations is forcing them to adopt all such methods and to undertake necessary activities to prevent further degradation of the environment. Reverse manufacturing is one of the popular methods undertaken by the manufacturing organizations to recycle the goods after these have been procured from the customers and their reuse effectively for the same purpose. Re-usable and recycle-able materials/articles are procured from the customers through reverse-distribution channels and reconverted through appropriate processes to appear as new and usable. This paper has been prepared in the backdrop of a very high level of ecological consciousness on the part of the government and society. Our research work also facilitates to include implication of research topics such as flexible manufacturing system. In the present consumerist society and a cut-throat competition in the market, the manufacturers are not only employing newer methods of distribution but also newer formats of

* Corresponding author.

E-mail: nancineha.saxena@gmail.com (N. Saxena)

(c) 2013 Growing Science Ltd. All rights reserved.

doi: $10.5267 /$ j.ijiec.2013.05.004 
distribution. The companies are entering rural markets, semi-urban areas and reaching out to the unexpected segments of potential customers. In addition to generate a spurt in demand, the companies are using innovative marketing strategies and innovative marketing tactics with varying degrees of effectiveness. As far as distribution is concerned new departmental stores, new shopping malls are sprouting up even in the unrepresented geographical areas. Because of all this, the visibility and reach of the brand/product has increased manifolds, which causes sudden fluctuations in demand. There is a strong need for a flexible manufacturing system, which can take care of the above realities and adjusts itself to the realities of the market.

For the past few decades, reverse logistics has been receiving much attention. Schrady (1967) first studied the problem on optimal lot sizes for production/procurement and recovery. For issues in the greening process, Nahmias and Rivera (1979) studied an EPQ variant of Schrady's model (1967) with a finite recovery rate. Richter (1996a, 1996b, 1997) and Richter and Dobos (1999) investigated a waste disposal model by considering the returned rate as a decision variable. Dobos and Richter $(2003,2004)$ investigated a production/remanufacturing system with constant demand that is satisfied by noninstantaneous production and remanufacturing for single and multiple remanufacturing and production cycle. Dobos and Richter (2006) extended their previous model and assumed that the quality of collected returned items is not always suitable for further repairing. Konstantaras and Skouri (2010) presented a model by considering a general cycle pattern in which a variable number of reproduction lots of equal size were followed by a variable number of manufacturing lots of equal size. They also studied a special case where shortages were allowed in each manufacturing and reproduction cycle and similar sufficient conditions, as the non-shortages case, are given.

El Saadany and Jaber (2010) extended the models developed by Dobos and Richter (2003, 2004) by assuming that the collection rate of returned items is dependent on the purchasing price and the acceptance quality level of these returns. That is, the flow of used/returned items increases as the purchasing price increases, and decreases as the corresponding acceptance quality level increases. Alamri (2010) developed a general reverse logistics inventory model. Chung and Wee (2011) developed an inventory model on short life-cycle deteriorating product remanufacturing in a green supply chain model. Singh and Saxena (2012) derived an optimal returned policy for a reverse logistics inventory model with backorders.

An increase in the shelf space can influence more customers. In this connection, the observations made by Levin et al. (1972) and Silver and Peterson (1985) should be mentioned. They observed that the presence of greater quantity of the same item tends to attract more customers. The reason behind this fact is a typical psychology of the customers. They may have the feeling of obtaining a wide range for selection when a large amount is stored/displayed.Gupta and Vrat (1986) developed models for stock dependent consumption rate. Mandal and Phaujdar (1989) developed an inventory model for deteriorating items and stock dependent consumption rate. Schweitzer and Seidmann (1991) established optimizing processing rate for flexible manufacturing systems. Giri and Chaudhuri (1998) developed deterministic model of perishable inventory with stock-dependent demand rate and nonlinear holding cost and proved that the non-linear holding cost affects the total average cost. Sana et al. (2004) established a production-inventory model for a deteriorating item with trended demand and shortages. Teng and Chang (2005) proposed economic production model for deteriorating item with price and stock dependent demand. Singh and Jain (2009) worked on reserve money for an EOQ model in an inflationary environment under supplier credits. Singh and Singh (2010) worked on supply chain model with stochastic lead-time under imprecise partially backlogging for expiring items. Singh et al. (2010) contributed on an inventory model for deteriorating items with shortages and stock-dependent demand under inflation for two-shops under one management. Yadav et al. (2012) developed an inventory model of deteriorating items with stock dependent demand using genetic algorithm in fuzzy environment. Singh et al. (2013) developed a supply chain inventory model for shortages with variable demand rate. 
This model consists of two systems forward manufacturing and reverse manufacturing. At the beginning of each cycle, the inventory is zero. The production starts at the very beginning of the cycle. As production progresses the inventory of finished goods piles up even after meeting the market demand, deterioration/obsolescence. At the beginning of each cycle, the process of collecting returnable items in a separate store also begins. At a point where the production from the forward manufacturing system stops; the collection process of returnable items also stops at the same point (For simplicity, we assume there is no collection of used items once the remanufacturing of collected items starts). At this very point the remanufacturing of reusable items begin at a constant rate. The accumulated inventory produced from the advanced manufacturing system in the meanwhile starts getting consumed and ultimately becomes nil. The accumulated inventory of remanufacturing products, which are assumed to be as good as the newly produced products is consumed when the shortages from the forward manufacturing system begin to surface. In addition, at this stage, there is no production and inventory of remanufactured items is consumed till it becomes nil. When the inventory of remanufactured items is also nil, inventory shortages begin to accumulate for some time. Thereafter, production starts and shortages are gradually cleared after meeting demand and the cycle ends with zero inventories. Geometrical description is shown in Fig 1.

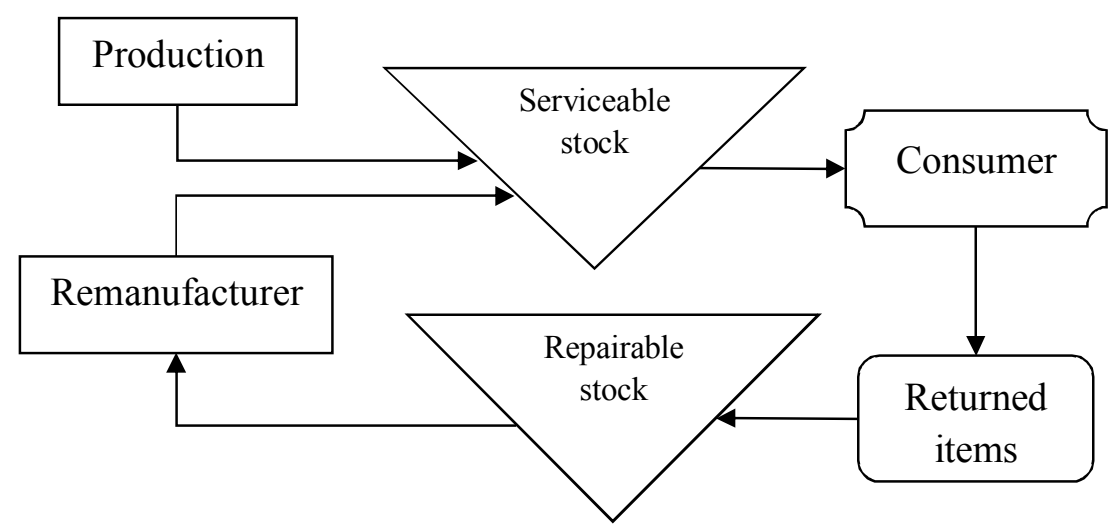

Fig. 1. Flow of inventory in the integrated supply system

\section{Assumptions \& notations}

1. Production rate is linear function of demand.

2. The demand rate is deterministic and is a known function of the on hand inventory q. The functional relationship between the demand rate $f(q)$ and the inventory level $q(t)$ is given by the following expression:

$$
f(q)=D q^{\beta}, D>0,0<\beta<1, q \geq 0
$$

Where $\beta$ denotes the shape parameter and is a measure of responsiveness of the demand to changes in the level of on hand inventory and D denotes the scale parameter.

3. Deterioration rate is constant.

4. Items are returnable and are remanufactured. Remanufactured items are as good as new ones and they are used during the shortage period of forward manufacturing.

5. The time horizon of the inventory system is infinite. Only a typical planning schedule of length $\mathrm{T}$ is considered, all remaining cycles are identical.

6. Shortages are allowed and are completely backlogged.

7. The production time interval for forward production coincides with the collection time interval for reverse manufacturing. (This assumption is not applicable during the period of shortages)

Notations for forward manufacturing system and reverse system: 
$q(t) \quad: \quad$ On hand inventory level at any time t.

$f(q): \quad$ Demand rate, $f(q)=D q^{\beta}, D>0,0<\beta<1$

$P \quad: \quad$ Production rate, $P=l f(q)$ where $l$ is a scale parameter, $P>f(q), l>1$

$K \quad: \quad$ Ordering cost per order.

$c_{h} \quad: \quad$ Holding cost per unit per unit of time during the forward manufacturing.

$c_{p} \quad: \quad$ Production cost per item.

$\theta \quad: \quad$ Deterioration rate.

$c_{h}^{\prime} \quad$ : Holding cost per unit per unit of time during the collecting and consuming process for the reverse manufacturing.

$c_{h}^{\prime \prime} \quad$ : Holding cost per unit per unit of time during the remanufacturing process for the reverse manufacturing.

$q_{c}\left(t_{c}\right)$ : Inventory level during the collecting process for the reverse manufacturing.

$q_{1}(t)$ : Inventory level during the remanufacturing process for the reverse manufacturing.

$\xi \quad: \quad$ Fraction of the production lot size $0<\xi<1$

$Q \quad$ : $\quad$ Maximum inventory level during forward manufacturing.

$R_{c} \quad: \quad$ Rate of collection of returnable items.

$M \quad$ : $\quad$ Rate of production of returnable items to be remanufactured.

$t_{p} \quad: \quad$ Time when production of forward manufacturing stops and also the time when collecting process for reverse manufacturing stops. At this very time remanufacturing of collected items start.

$t_{s} \quad: \quad$ Time when remanufacturing of returnable items stops and also the time when accumulated inventory of forward manufacturing vanishes.

$t_{s_{1}} \quad: \quad$ Time when accumulated remanufactured inventory vanishes and shortages start.

$t_{m} \quad: \quad$ Time when production starts again during the period of shortage.

$T^{\prime} \quad$ : $\quad$ Time to complete cycle.

$S_{1} \quad: \quad$ Maximum inventory level of remanufactured items.

$S^{\prime} \quad$ : $\quad$ Maximum shortages.

$c_{p}^{\prime} \quad: \quad$ Cost of purchasing the returnable items per unit.

$c_{p}^{\prime \prime} \quad: \quad$ Production cost of remanufactured items per unit.

$c_{s} \quad: \quad$ Shortage cost per unit per unit of time.

\section{Mathematical modeling}

There are five stages in the Model (in each cycle as represented in the figure). The governing differential equations are as below:

Forward manufacturing process

$$
\begin{array}{lll}
\frac{d q}{d t}+\theta q=(l-1) D q^{\beta} & q(0)=0, & 0 \leq t \leq t_{p} \\
\frac{d q}{d t}+\theta q=-D q^{\beta} & q\left(t_{p}\right)=Q & t_{p} \leq t \leq t_{s}
\end{array}
$$

Differential equations representing reverse manufacturing collecting time $\&$ consuming time. 
$\begin{array}{lrl}\frac{d q_{c}\left(t_{c}\right)}{d t_{c}}=R_{c}-\theta q_{c}\left(t_{c}\right) & q_{c}(0)=0 & 0 \leq t_{c} \leq t_{p} \\ \frac{d q_{c}\left(t_{c}\right)}{d t_{c}}=-M-\theta q_{c}\left(t_{c}\right) & q_{c}\left(t_{p}\right)=B \xi & t_{p} \leq t_{c} \leq t_{s}\end{array}$

where $\mathrm{B}=(1-\theta) l D\left(\frac{Q}{(l-1) D}+\frac{\theta Q^{\alpha+1}}{(l-1)^{2} D^{2}(\alpha+1)}\right)$ is the production lot size during the interval $\left[0, t_{p}\right]$ in forward manufacturing system (see Appendix A)

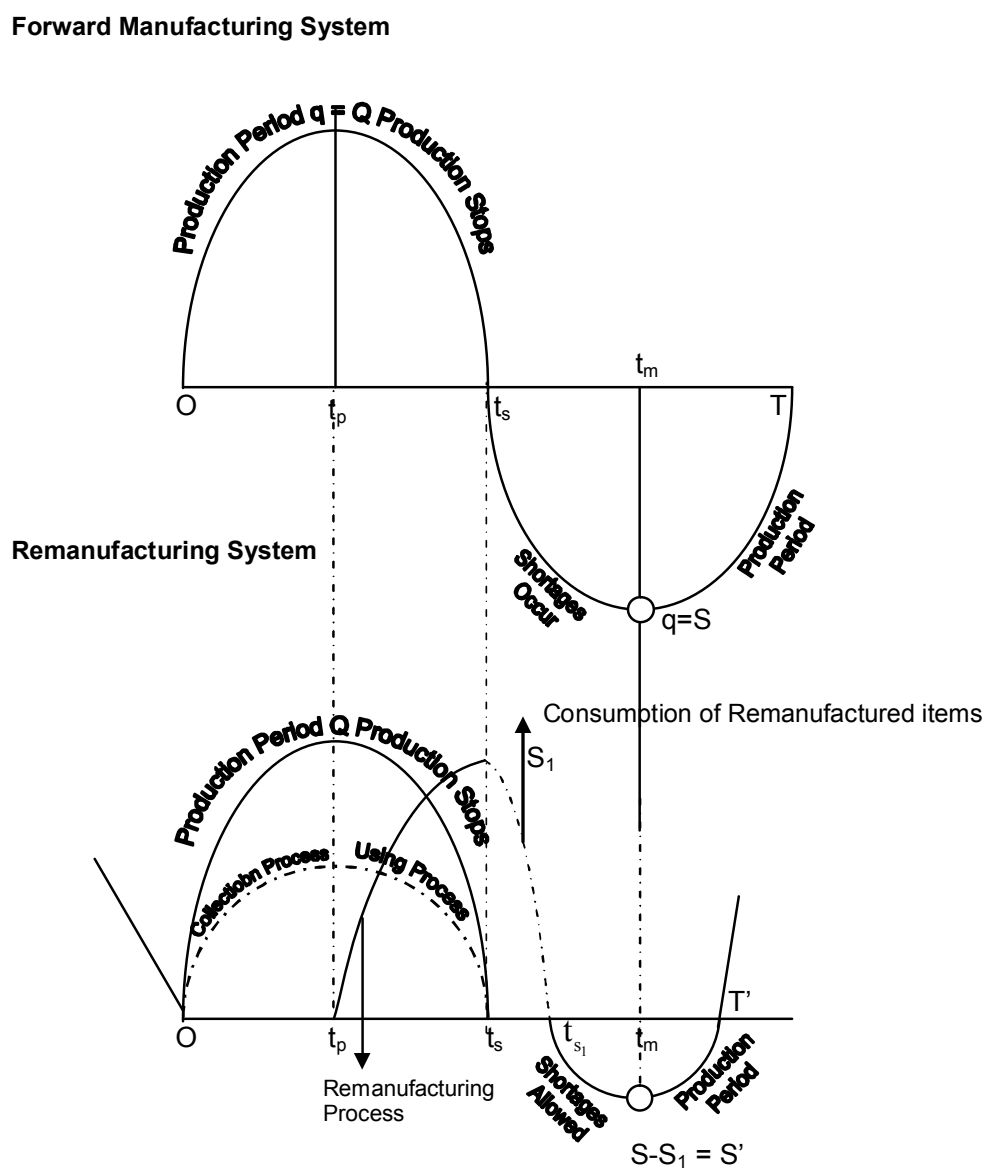

Fig. 1. Flow of inventory

Differential equations representing inventory of remanufactured items.
$\frac{d q_{1}}{d t}=M-\theta q_{1}$
$q_{1}\left(t_{p}\right)=0$
$t_{p} \leq t \leq t_{s}$
$\frac{d q}{d t}+\theta q(t)=-D q^{\beta}$
$q\left(t_{s}\right)=S_{1}$
$t_{s} \leq t \leq t_{s_{1}}$
$\frac{d q}{d t}=-D q^{\beta}$
$q\left(t_{s_{1}}\right)=0$
$t_{s_{1}} \leq t \leq t_{m}$
$\frac{d q}{d t}+\theta q(t)=(l-1) D q^{\beta}$
$q\left(t_{m}\right)=S^{\prime}=S-S_{1}$
$t_{m} \leq t \leq T^{\prime}$

$3(\mathrm{c})$

Solving Eq. (1a) and Eq. (1b), we have 


$$
\begin{array}{ll}
t=\left(\frac{q^{\alpha}}{(l-1) \alpha D}+\frac{\theta q^{2 \alpha}}{2(l-1)^{2} \alpha D^{2}}+--\right) & 0 \leq t \leq t_{p} \\
q^{\alpha} e^{\theta \alpha t}=\frac{-D}{\theta} e^{\theta \alpha t}+\left(\frac{D}{\theta}+Q^{\alpha}\right) e^{\theta \alpha t p} & t_{p} \leq t \leq t_{s}
\end{array}
$$

Solving Eq. (2a) and Eq. (2b)

$$
\begin{array}{ll}
q_{c}=\frac{R_{c}}{\theta}\left(1-e^{-\theta t_{c}}\right) & 0 \leq t_{c} \leq t_{p} \\
q_{c}=-\frac{M}{\theta}+\left(\frac{M}{\theta}+B \xi\right) e^{\theta\left(t_{p}-t_{c}\right)} & t_{p} \leq t_{c} \leq t_{s}
\end{array}
$$

Now to find holding cost for inventory of collected items during interval $\left[0, t_{s}\right]$, we have

$$
\begin{aligned}
& =c_{h}^{\prime} \int_{0}^{t_{p}} q_{c}\left(t_{c}\right) d t_{c}+c_{h}^{\prime} \int_{t_{p}}^{t_{s}} q_{c}\left(t_{c}\right) d t_{c} \\
& =c_{h}^{\prime}\left(\frac{B^{2} \xi^{2}}{2 R_{c}}+\frac{B^{3} \xi^{3} \theta}{3 R_{c}^{2}}\right)+c_{h}^{\prime}\left(\frac{B^{2} \xi^{2}}{2 M}-\frac{B^{3} \xi^{3} \theta}{3 M^{2}}\right) \text { (See Appendix C) }
\end{aligned}
$$

$\therefore$ Total cost of collected items during $\left[0, t_{s}\right]$ (Holding cost + Deterioration cost)

$$
=\left(c_{h}^{\prime}+\theta c_{p}^{\prime}\right)\left(\frac{B^{2} \xi^{2}}{2}\left(\frac{1}{R_{c}}+\frac{1}{M}\right)+\frac{B^{3} \xi^{2} \theta}{3}\left(\frac{1}{R_{c}^{2}}-\frac{1}{M^{2}}\right)\right)
$$

Inventory of Remanufactured items

Solving (3a),(3b), (3c) \& (3d) and using boundary conditions

$$
\begin{array}{ll}
q_{1}=\frac{M}{\theta}\left(1-e^{\theta\left(t_{p}-t\right)}\right) & t_{p} \leq t \leq t_{s} \\
q^{\alpha}=-\frac{D}{\theta}+\left(S_{1}^{\alpha}+\frac{D}{\theta}\right) e^{\theta \alpha\left(t_{s}-t\right)} & t_{s} \leq t \leq t_{s_{1}} \\
q^{\alpha}=D \alpha\left(t_{s_{1}}-t\right) & t_{s_{1}} \leq t \leq t_{m} \\
q^{\alpha}=\frac{(l-1) D}{\theta}+\left(S^{\prime \alpha}-\frac{(l-1)}{\theta} D\right) e^{\theta \alpha\left(t_{m}-t\right)} & t_{m} \leq t \leq T^{\prime}
\end{array}
$$

The cycle consists of five stages; time for each stage and the cycle time have been calculated as below:

$$
\begin{gathered}
t_{p}=\frac{Q^{\alpha}}{(l-1) D \alpha}\left(1+\frac{\theta Q^{\alpha}}{2(l-1) D}\right) \quad t_{s}=t_{p}+\frac{Q^{\alpha}}{D \alpha}\left(1-\frac{\theta Q^{\alpha}}{2 D}\right) \quad t_{s_{1}}=t_{s}+\frac{S_{1}^{\alpha}}{D \alpha}-\frac{\theta S_{1}^{2 \alpha}}{2 D^{2} \alpha} \quad t_{m}=t_{s_{1}}-\frac{S^{\prime \alpha}}{D \alpha} \\
T^{\prime}=\frac{Q^{\alpha}}{(l-1) D \alpha}\left(1+\frac{\theta Q^{\alpha}}{2(l-1) D}\right)+\frac{Q^{\alpha}}{D \alpha}\left(1-\frac{\theta Q^{\alpha}}{2 D}\right)+\frac{S_{1}^{\alpha}}{D \alpha}-\frac{\theta S_{1}^{2 \alpha}}{2 D^{2} \alpha}+\frac{l}{(1-l) D \alpha} S^{\prime \alpha}-\frac{S^{\prime 2 \alpha} \theta}{2(1-l)^{2} D^{2} \alpha}
\end{gathered}
$$

The above expression represents time to complete one cycle.

Inventory of remanufactured items during interval $\left(t_{p} \leq t \leq t_{s}\right)$

$$
=\int_{t_{p}}^{t_{s}} q_{1} d t \quad=\int_{0}^{S_{1}} q_{1}\left(\frac{1}{M}+\frac{\theta q_{1}}{M^{2}}\right) d q_{1}=\frac{S_{1}^{2}}{2 M}+\frac{\theta S_{1}^{3}}{3 M^{2}}
$$

Total cost of remanufactured inventory (Holding cost + Deterioration cost) 


$$
=\left(c_{h}^{\prime \prime}+\theta c_{p}^{\prime \prime}\right)\left(\frac{\theta S_{1}^{3}}{3 M^{2}}+\frac{S_{1}^{2}}{2 M}\right) \quad \text { where } t_{s}-t_{p}=\frac{S_{1}}{M}+\frac{\theta S_{1}^{2}}{2 M^{2}}
$$

(See Appendix D to find the relation to find $\mathrm{S}_{1}$ at time $t_{s}$ ).

In forward manufacturing system, period of shortage starts at $\mathrm{t}=t_{s}$. It has been assumed that remanufactured items are as good as the new ones and they are used during the shortage period of forward manufacturing.

Holding cost in interval $\left[t_{s}, t_{s_{1}}\right]$

$$
\begin{gathered}
=c_{h} \int_{t_{s}}^{t_{s 1}} q d t=c_{h} \int_{S_{1}}^{0} q\left(-\frac{q^{\alpha-1}}{D}+\frac{\theta q^{2 \alpha-1}}{D^{2}}\right) d q(\text { See Appendix E) } \\
h c=c_{h}\left(\frac{S_{1}^{\alpha+1}}{D(\alpha+1)}-\frac{\theta S_{1}^{2 \alpha+1}}{D^{2}(2 \alpha+1)}\right)
\end{gathered}
$$

Total cost of remanufactured inventory (i.e. holding cost + deterioration cost) in interval $\left[t_{s}, t_{s_{1}}\right]$

$$
=\left(c_{h}+\theta c_{p}\right)\left(\frac{S_{1}^{\alpha+1}}{D(\alpha+1)}-\frac{\theta S_{1}^{2 \alpha+1}}{D^{2}(2 \alpha+1)}\right)
$$

Shortage cost in $\left[t_{s_{1}}, t_{m}\right]$

$$
=-c_{s} \int_{t_{s_{1}}}^{t_{m}} q d t \quad=-c_{s} \int_{o}^{S^{\prime}}-\frac{q^{\alpha}}{D} d q=\frac{c_{s} S^{\prime \alpha+1}}{D(\alpha+1)}
$$

Shortage cost in $\left(t_{m} \leq t \leq T^{\prime}\right)$ (see Appendix F)

$$
=-c_{s} \int_{S^{\prime}}^{0} q\left(\frac{q^{\alpha-1}}{(l-1) D}+\frac{\theta q^{2 \alpha-1}}{(l-1)^{2} D^{2}}\right) d q=c_{s}\left(\frac{S^{\prime \alpha+1}}{(l-1) D(\alpha+1)}+\frac{\theta S^{\prime 2 \alpha+1}}{(l-1)^{2} D^{2}(2 \alpha+1)}\right)
$$

This represents shortage cost in $\left[t_{m}, T^{\prime}\right]$

Total shortage cost

$$
=s c \text { in }\left[t_{s_{1}}, t_{m}\right]+\operatorname{sc} \text { in }\left[t_{m}, T^{\prime}\right]=\frac{c_{s} S^{\prime \alpha+1}}{D(\alpha+1)}+\frac{c_{s} S^{\prime \alpha+1}}{(l-1) D(\alpha+1)}+\frac{c_{s} \theta S^{\prime 2 \alpha+1}}{(l-1)^{2} D^{2}(2 \alpha+1)}
$$

Total holding cost and deterioration cost in interval $\left[0, T^{\prime}\right]$ (Forward system + Reverse System) $\mathrm{HC}+\mathrm{DC}=\operatorname{cost}$ in $\left[0, t_{p}\right]+\operatorname{cost}$ in $\left[t_{p}, t_{s}\right]+\operatorname{cost}$ in $\left[t_{s}, t_{s_{1}}\right]$

$$
\begin{aligned}
= & \frac{\left(c_{h}+\theta c_{p}\right)}{(l-1) D}\left[\frac{Q^{\alpha+1}}{\alpha+1}+\frac{\theta Q^{2 \alpha+1}}{(2 \alpha+1)(l-1) D}\right]+\left(c_{h}+\theta c_{p}\right) \times\left[\frac{Q^{\alpha+1}}{D(\alpha+1)}-\frac{\theta Q^{2 \alpha+1}}{D^{2}(2 \alpha+1)}\right] \\
& +\left(c_{h}^{\prime}+\theta c_{p}^{\prime}\right)\left(\frac{B^{2} \xi^{2}}{2}\left(\frac{1}{R_{c}}+\frac{1}{M}\right)+\frac{B^{3} \xi^{3} \theta}{3}\left(\frac{1}{R_{c}^{2}}-\frac{1}{M^{2}}\right)\right) \\
& +\left(c_{h}^{\prime \prime}+\theta c_{p}^{\prime \prime}\right)\left(\frac{\theta S_{1}^{3}}{3 M^{2}}+\frac{S_{1}^{2}}{2 M}\right)+\left(c_{h}+\theta c_{p}\right)\left(\frac{S_{1}^{\alpha+1}}{D(\alpha+1)}-\frac{\theta S_{1}^{2 \alpha+1}}{D^{2}(2 \alpha+1)}\right)
\end{aligned}
$$

The total inventory cost per unit time is therefore given by

$$
T A C\left(Q, S^{\prime}\right)=\frac{K+H C+D C+S C}{T} .
$$

Our problem is to find the time to stop the production when $\mathrm{q}$ takes optimum value $\mathrm{Q}$ and the time to again start the production when maximum shortages accumulate. 
$\frac{\partial}{\partial Q}(T A C)=0, \frac{\partial}{\partial S^{\prime}}(T A C)=0, \frac{\partial(T A C)}{\partial S^{\prime}}=0 \Rightarrow T^{\prime}\left[\frac{\partial S C}{\partial S^{\prime}}+\frac{\partial}{\partial S^{\prime}} D C\right]-(K+H C+D C+S C) \frac{\partial T^{\prime}}{\partial S^{\prime}}=0$

Also $T^{\prime}\left[\frac{\partial H C}{\partial Q}+\frac{\partial D C}{\partial Q}\right]-(K+H C+D C+S C) \frac{\partial T}{\partial Q}=0$

As $\theta \rightarrow 0, \alpha \rightarrow 1$, we have

$$
\frac{\frac{\partial S C}{\partial S^{\prime}}}{\frac{\partial H C}{\partial Q}}=\frac{\frac{\partial T^{\prime}}{\partial S^{\prime}}}{\frac{\partial T^{\prime}}{\partial Q}}
$$

As $\theta \rightarrow 0, \alpha \rightarrow 1$

$T^{\prime}=\frac{Q l}{(l-1) D}+\frac{l}{(1-l) D} S^{\prime}+\frac{S_{1}}{D}$

Using Appendix D, As $\theta \rightarrow 0 \quad S_{1} \rightarrow M\left(t_{s}-t_{p}\right)$

and using relation $\frac{B \xi}{M}=t_{s}-t_{p} \quad S_{1} \rightarrow B \xi$

and also $\mathrm{B} \rightarrow \frac{Q l}{l-1} \therefore S_{1} \rightarrow \frac{Q l \xi}{l-1}=Q \eta($ say $)$

$\therefore T^{\prime}=\frac{Q l}{(l-1) D}+\frac{l}{(1-l) D} S^{\prime}+\frac{Q l \xi}{(l-1) D}$

(Also as $\xi \rightarrow 0$ i.e. there is no remanufacturing)

[Than $T^{\prime} \rightarrow T$ which is time to complete the cycle in forward manufacturing when reverse manufacturing is not included.]

As $\theta \rightarrow 0, \alpha \rightarrow 1$, we have $\mathrm{DC}=0$ and

$\mathrm{HC}=\frac{c_{h} l Q^{2}}{2(l-1) D}+c_{h}^{\prime} \frac{B^{2} \xi^{2}}{2 R_{c}}+c_{h}^{\prime \prime} \frac{S_{1}^{2}}{2 M}+c_{h} \frac{S_{1}^{2}}{2 D}+\frac{B^{2} \xi^{2} c_{h}^{\prime}}{2 M}$

and also $S_{1} \rightarrow B \xi$

$\mathrm{HC}=\frac{c_{h} l Q^{2}}{2(l-1) D}+c_{h} \cdot \frac{B^{2} \xi^{2}}{2 R_{c}}+c_{h}^{\prime \prime} \frac{B^{2} \xi^{2}}{2 M}+c_{h} \frac{B^{2} \xi^{2}}{2 D}+\frac{B^{2} \xi^{2} c_{h}{ }^{\prime}}{2 M}$

As $\theta \rightarrow 0, \alpha \rightarrow 1$

$\mathrm{SC}=\frac{c_{S} S^{\prime 2} l}{2(l-1) D}$

Substituting all the above values in relation (R1)

$\frac{\frac{c_{S} S^{\prime} l}{(l-1) D}}{\frac{c_{h} l Q}{(l-1) D}+\frac{c_{h}^{\prime} Q \eta^{2}}{R_{c}}+\frac{c_{h}^{\prime \prime} Q \eta^{2}}{M}+\frac{c_{h} Q \eta^{2}}{D}+\frac{Q \eta^{2} c_{h}^{\prime}}{M}}=\frac{l /(1-l) D}{\frac{l}{(l-1) D}+\frac{\eta}{D}} \quad$ where $\eta=\frac{l \xi}{l-1}$

[As $\xi \rightarrow 0$, we get $c_{s} S=-c_{h} Q$, ]. If we assume holding cost per unit per unit of time remains same during forward and reverse manufacturing i.e. $c_{h}=c_{h}^{\prime}=c_{h}^{\prime \prime}$, We have

$$
\frac{c_{s} S^{\prime}}{\frac{c_{h} l Q}{l-1}\left(\frac{1}{D}+\frac{l}{(l-1)} \xi^{2}\left(\frac{1}{R_{C}}+\frac{2}{M}+\frac{1}{D}\right)\right)}=\frac{-1}{\frac{l}{(l-1) D}(1+\xi)}
$$


$\Rightarrow c_{s} S^{\prime}(1+\xi)=-c_{h} Q(1+\eta \xi \zeta D) \quad$ Which gives relation in $S^{\prime}$ and $Q$.

Substituting all the values in (R1)

As $\theta \rightarrow 0, \alpha \rightarrow 1$

$$
\frac{2 k(l-1) D}{l}=Q^{2}(1+\eta \xi \zeta D) c_{h}\left(1+\frac{c_{h}(1+\eta \xi \zeta D)}{(1+\xi)^{2} c_{s}}\right)
$$

As $\xi \rightarrow 0 Q^{*}=\sqrt{\frac{2 k(l-1) D c_{S}}{l c_{h}\left(c_{s}+c_{h}\right)}}$

As $l$ increases, production occurs at a more rapid rate. Hence for large $l$, the model should approach the instantaneous delivery situation of the EOQ model. For large $l, 1-\frac{1}{l} \rightarrow 1$. Thus as $l$ increases towards infinity, the optimal run size for the model approaches the EOQ when shortages are allowed.

\section{Numerical example}

The above theoretical results are illustrated through the numerical verification. Here we are presenting the computational results obtained using Newton Raphson Method which give insight about the behavior of optimal run size $\mathrm{Q}^{*}$, production cycle time $T^{\prime}$ and the effects of reverse manufacturing on the total average cost TAC. To illustrate the proposed model, we have considered the following input parameters in appropriate units

$D=2.0, c_{h}=c_{h^{\prime}}=c_{h^{\prime \prime}}=0.5, K=200, l=2, c_{s}=0.5$ and as we have taken in the last section $\theta \rightarrow 0$.

Here we derive the optimal solution for the different returned rate and holding cost. Results are presented in Tables 1 as follows,

Table 1

Effects of $\xi$ on $\left(\mathrm{Q}^{*}, T^{\prime}, \mathrm{TAC}\right)$

\begin{tabular}{cccccc}
\hline$\xi$ & 0.2 & 0.4 & 0.6 & 0.8 & 1 \\
$Q^{*}$ & 14.825 & 11.785 & 9.7823 & 8.3621 & 7.303 \\
$T$ & 38.54 & 37.712 & 37.173 & 36.792 & 36.516 \\
Average HC & 2.3952 & 2.302 & 2.266 & 2.2239 & 2.192 \\
Average SC & 2.793 & 2.983 & 3.115 & 3.2124 & 3.288 \\
\hline TAC & 10.38 & 10.606 & 10.7605 & 10.8720 & 10.954 \\
\hline
\end{tabular}

Table 2

Effects of holding cost on $\left(\mathrm{Q}^{*}, T^{\prime}, \mathrm{TAC}\right)$

\begin{tabular}{llllll}
\hline$c_{h}$ & 0.2 & 0.4 & 0.6 & 0.8 & 1 \\
$Q^{*}$ & 14.433 & 8.704 & 6.29 & 4.950 & 4.082 \\
$T$ & 46.186 & 38.298 & 35.22 & 33.66 & 32.64 \\
Average HC & 2.706231 & 2.3738 & 2.022013 & 1.74706 & 1.53 \\
Average SC & 1.6238 & 2.8487 & 3.6383 & 4.193 & 4.59 \\
TAC & 8.6603 & 8.66084 & 8.6467 & 8.6593 & 8.655 \\
\hline
\end{tabular}

\subsection{Observations}

Following observations are made from Table 1: 
- As $\xi$ increases, Q and $T^{\prime}$ decreases and holding cost also decreases.

- As $\xi$ increases, shortage cost increases and total average cost slightly increase.

Observations made from Table2:

- Case 1: When holding cost per unit is lesser than the shortage cost per unit (0.5)

(a) As holding cost increases, Q \& $T^{\prime}$ decreases.

(b) As holding cost increases, there is very slight increase in the total average cost.

- Case 2:When holding cost per unit is greater than the shortage cost per unit (0.5)

(a) As holding cost increases, Q \& $T^{\prime}$ decreases but the rate of decreasing is less as compared to Case 1.

(b) As holding cost increases, total average cost increases \& the rate of increasing is more as compared to Case 1.

Comparative observations from Table 1 and Table 2

- When $c_{h}=c_{s}$, total average cost incurred is more as compared to the cost incurred when $c_{h} \neq c_{s}$.

\section{Conclusion}

When remanufacturing is undertaken, from the management standpoint there is no perceptible cost difference in terms of total average cost consisting of holding cost, shortage cost, deterioration cost \& set-up cost. In view of the governments concern about ecological protection, the management can adopt the system at almost no major incremental costs. As the ratio $\xi$ increases, there is very slight increase in the total average cost. Further research can be extended to consider the issue of multi objective optimization model, collection of used items during reverse manufacturing period also, inflation and discounting etc.

\section{Appendix A}

$\mathrm{B}=$ Production lot size during forward manufacturing system $=$ Production - deterioration

$$
=\int_{0}^{t p} P d t-\int_{0}^{t p} \theta P d t=(1-\theta) \int_{0}^{t p} l D q^{\beta} d t
$$

Using (1as), we have, $t=\frac{q^{\alpha}}{(l-1) D \alpha}+\frac{\theta q^{2 \alpha}}{2(l-1)^{2} D^{2} \alpha}$

Using in (i)

$$
B=(1-\theta) \int_{0}^{Q} l D\left(\frac{q^{\beta+\alpha-1}}{(l-1) D}+\frac{\theta q^{\beta+2 \alpha-1}}{(l-1)^{2} D^{2}}\right) d q=(1-\theta) l D\left(\frac{Q}{(l-1) D}+\frac{\theta Q^{\alpha+1}}{(l-1)^{2} D^{2}(\alpha+1)}\right)
$$

As $\theta \rightarrow 0, B \rightarrow \frac{Q l}{l-1}$

\section{Appendix B}

Using $q_{c}\left(t_{p}\right)=B \xi$ in $q_{c}=\frac{R_{c}}{\theta}\left(1-e^{-\theta t_{c}}\right) \quad B \xi=\frac{R_{c}}{\theta}\left(\theta t_{p}-\frac{\theta^{2} t_{p}^{2}}{2}\right)$

$$
\text { As } \theta \rightarrow 0 \quad B \xi \rightarrow R_{c} t_{p} \quad \Rightarrow R_{c}=\frac{B \xi}{t_{p}}
$$

This relation can be used to find the rate of collection. 


\section{Appendix C}

$\int_{0}^{t_{p}} q_{c}\left(t_{c}\right) d t_{c}=\int_{0}^{B \xi}\left(\frac{q_{c}}{R_{c}}+\frac{q_{c}^{2} \theta}{R_{c}^{2}}\right) d q_{c}$

$$
\left.\begin{array}{l}
t_{c}=\frac{q_{c}}{R_{c}}+\frac{q_{c}^{2} \theta}{2 R_{c}{ }^{2}} \\
d t_{c}=\left(\frac{1}{R_{c}}+\frac{q_{c} \theta}{R_{c}{ }^{2}}\right) d q_{c}
\end{array}\right]
$$

Similarly $\int_{t_{p}}^{t_{s}} q_{c}\left(t_{c}\right) d t_{c}=\int_{B \xi}^{0}\left(-\frac{q_{c}}{M}+\frac{\theta q_{c}^{2}}{M^{2}}\right) d q_{c}$

Using (2bs), we have $\frac{q_{c}+\frac{M}{\theta}}{\frac{M}{\theta}+B \xi}=e^{\theta\left(t_{p}-t_{c}\right)} \Rightarrow \theta\left(t_{p}-t_{c}\right)=\log \left(1+\frac{\theta q_{c}}{M}\right)-\log \left(1+\frac{\theta B \xi}{M}\right)$

$t_{c}=t_{p}-\frac{q_{c}}{M}+\frac{\theta q_{c}^{2}}{2 M^{2}}+\frac{B \xi}{M}-\frac{B^{2} \xi^{2} \theta}{2 M^{2}} \quad d t_{c}=\left(-\frac{1}{M}+\frac{\theta q_{c}}{M^{2}}\right) d q_{c}$

Using $q_{c}\left(t_{s}\right)=0$ in equation (2bs)

$$
-\log \left(1+\frac{B \xi \theta}{M}\right)=\theta\left(t_{p}-t_{s}\right) \quad \frac{B \xi \theta}{M}-\frac{B^{2} \xi^{2} \theta^{2}}{2 M^{2}}=\theta\left(t_{s}-t_{p}\right)
$$

This relation can be used to find the rate of production of remanufactured products.

\section{Appendix D}

$q_{1}=\frac{M}{\theta}\left(1-e^{\theta\left(t_{p}-t\right)}\right) \Rightarrow t=t_{p}+\frac{q_{1}}{M}+\frac{\theta q_{1}^{2}}{2 M^{2}}$

$q_{1}\left(t_{s}\right)=S_{1}$

$$
t_{s}=t_{p}+\frac{S_{1}}{M}+\frac{\theta S_{1}^{2}}{2 M^{2}}
$$

$$
t_{s}-t_{p}=\frac{S_{1}}{M}+\frac{\theta S_{1}^{2}}{2 M^{2}}
$$

As $\theta \rightarrow 0, S_{1} \rightarrow M\left(t_{s}-t_{p}\right)$

The above relation can be used to find the amount of inventory at time $t=t_{s}$.

\section{Appendix E}

$$
\begin{aligned}
& e^{\theta \alpha\left(t_{s}-t\right)}=\frac{q^{\alpha}+\frac{D}{\theta}}{S_{1}^{\alpha}+\frac{D}{\theta}} \quad \theta \alpha\left(t_{s}-t\right)=\log \left(1+\frac{\theta}{D} q^{\alpha}\right)-\log \left(1+\frac{\theta}{D} S_{1}^{\alpha}\right) \quad t=t_{s}-\frac{q^{\alpha}}{D \alpha}+\frac{\theta q^{2 \alpha}}{2 D^{2} \alpha}+\frac{S_{1}^{\alpha}}{D \alpha}-\frac{\theta^{2} S_{1}^{2 \alpha}}{2 D^{2} \alpha} \\
& d t=\left(-\frac{\alpha q^{\alpha-1}}{D \alpha}+\frac{2 \theta \alpha q^{2 \alpha-1}}{2 D^{2} \alpha}\right) d q
\end{aligned}
$$

\section{Appendix F}

In interval $\left(t_{m} \leq t \leq T^{\prime}\right)$

$$
\begin{aligned}
& q^{\alpha}=\frac{(l-1) D}{\theta}+\left(S^{\prime \alpha}-\frac{(l-1) D}{\theta}\right) e^{\theta \alpha\left(t_{m}-t\right)} \theta \alpha\left(t_{m}-t\right)=\log \left(1-\frac{\theta q^{\alpha}}{(l-1) D}\right)-\log \left(1-\frac{\theta S^{\prime \alpha}}{(l-1) D}\right) \\
& t-t_{m}=\frac{q^{\alpha}}{(l-1) D \alpha}+\frac{\theta q^{2 \alpha}}{2(l-1)^{2} D^{2} \alpha}+\frac{S^{\prime \alpha}}{(l-1) D \alpha}+\frac{\theta S^{\prime 2 \alpha}}{2(l-1)^{2} D^{2} \alpha}
\end{aligned}
$$

\section{References}

Alamri, A.A. (2010). Theory and methodology on the global optimal solution to a Reverse logistics inventory model for deteriorating items and time varying rates. Computer \& Industrial Engineering, (60), 236-247. 
Chung, C. J., \& Wee, H. M. (2011). Short life-cycle deteriorating product remanufacturing in a green supply chain inventory control system. International Journal of Production Economics, 129(1), 195-203.

Dobos, I., \& Richter, K. (2003). A production/recycling model with stationary demand and return rates. Central European journal of Operations Research, 11(1), 35-46.

Dobos, I., \& Richter, K. (2004). An extended production/recycling model with stationary demand and return rates. International Journal of production Economics, 90(3), 311-323.

Dobos, I., \& Richter, K. (2006). A production/recycling model with quality considerations. International Journal of Production Economics, 104(2), 571-579.

El Saadany A. M. A., \& Jaber M. Y. (2010). A production/remanufacturing inventory model with price and quality dependant return rate. Computers and Industrial Engineering, 58(3), 352-362.

Giri, B. C. \& Chaudhuri, K. S. (1998). Deterministic model of perishable inventory with stock-dependent demand rate and nonlinear holding cost. European Journal of Operations Research, 105, 467-474.

Gupta, R., \& Vrat, P. (1986). Inventory models for stock dependent consumption rate. Opsearch, 23, 19-24.

Konstantaras, I., \& Skouri, K. (2010). Lot sizing for a single product recovery system with variable set up numbers. European Journal of Operations Research, 203(2), 326-335.

Levin, R. I., Mclaughlin, C. P., Lamone, R. P., \& Kottas, J. F. (1972). Production, Operations Management: Contemporary Policy for Managing Operation System. (Newyork: McGraw-hill), 373.

Mandal, B.N., \& Phaujdar, S. (1989). An inventory model for deteriorating items and stock dependent consumption rate. Journal Operational Research Society, 40, 483-488.

Nahmias, N., \& Rivera, H. (1979). A deterministic model for a repairable item inventory system with a finite repair rate. International Journal of Production Research, 17 (3), 215-221

Richter, K. (1996). The EOQ repair and waste disposal model with variable setup numbers. European Journal of Operational Research, 95(2), 313-324.

Richter, K, (1996b). The extended EOQ repair and waste disposal model. International Journal of Production Economics, 45(1-3), 443-447.

Richter, K., \& Dobos, I. (1999). Analysis of the EOQ repair and waste disposal model with integer set up numbers. International journal of production economics, 59(1-3), 463-467.

Sana, S., Goyal, S.K., Chaudhuri K.S. (2004). A production-inventory model for a deteriorating item with trended demand and shortages. European Journal of Operational Research, 157 (2), 357-371.

Schrady, D.A. (1967). A deterministic inventory model for repairable items. Naval Research Logistics Quarterly, 14, 391-398.

Schweitzer, P. J., \& Seidmann, A. (1991). Optimizing, processing rate for flexible manufacturing systems. Management Science, 37, 454-466.

Silver, E. A., \& Peterson, R. (1985). Decision systems for inventory management and production planning, $2^{\text {nd }}$ ed. (Newyork: Wiley).

Singh, S.R., Gupta, V., \& Gupta, P. (2013). Three stage supply chain model with two warehouse, imperfect production, variable demand rate and inflation. International Journal of Industrial Engineering Computations, 4, 81-92.

Singh, S.R., \& Jain R. (2009). On reserve money for an EOQ model in an inflationary environment under supplier credits. Opsearch, 46 (2), 352-369.

Singh, S. R., Kumar N., \& Kumari, R. (2010). An inventory model for deteriorating items with shortages and stock-dependent demand under inflation for two-shops under one management. Opsearch, 47 (4), 311-329.

Singh, S. R., \& Saxena N. (2012). An Optimal Returned Policy for a Reverse Logistics Inventory Model with Backorders. Advances in Decision Sciences, vol. 2012, Article ID 386598, 21 pages.

Singh, S. R. \& Singh C. (2010). Supply chain model with stochastic lead time under imprecise partially backlogging and fuzzy ramp-type demand for expiring items. International Journal of Operational Research, 8(4), 511 - 522.

Teng, J. T. \& Chang, C. T. (2005). Economic production quantity models for deteriorating items with price and stock-dependent demand. Computational Operations Research, 32 (2), 297-308.

Yadav, D., Singh, S. R., \& Kumari R. (2012). Inventory model of deteriorating items with two-warehouse and stock dependent demand using genetic algorithm in fuzzy environment. Yugoslav Journal of Operations Research, 22 (1), 51-78. 